\title{
Multivessel coronary thrombosis, acute myocardial infarction, and no reflow in a patient with essential thrombocythaemia
}

\author{
H Terada, H Satoh, A Uehara
}

\begin{abstract}
Essential thrombocythaemia (ET) has been reported rarely to cause coronary thrombosis, but the management is still undefined. A 63 year old woman with multivessel coronary thrombosis, acute myocardial infarction (MI), and no reflow in reperfused coronary artery in association with ET is presented. The patient's platelet count was only moderately raised at the onset of MI, but peripheral blood smear and bone marrow evaluation revealed clumping giant platelets and numerous large hyperploid megakaryocytes. Long term prophylaxis with antiplatelet agents in patients with ET is recommended, even if the platelet count is not largely raised. Cytoreductive treatment may also be effective for secondary prevention when thrombotic complications occur.

(Heart 2000;83:e10)
\end{abstract}

Keywords: essential thrombocythaemia; thrombosis; coronary artery; no reflow

Essential thrombocythaemia (ET) is an acquired myeloproliferative disorder characterised by a consistently high platelet count and clinical tendencies to both haemorrhage and thrombosis. Fewer than 20 cases of angina pectoris and myocardial infarction (MI) have been reported in association with $\mathrm{ET}^{1-6}$ We describe a case of ET presenting with multivessel coronary thrombosis, acute MI, and no reflow in reperfused artery.

Division of Cardiology, Third Department of Internal Medicine, Hamamatsu

University School of

Medicine, 3600

Handa-cho,

Hamamatsu 431-3192, Japan

H Terada

H Satoh

A Uehara

Correspondence to:

Dr Satoh

email:

satoh36@hama-med.ac.jp

Accepted 6 January 2000 atrial fibrillation, or family history of coronary artery disease, but was mildly obese (body mass index $28.8 \mathrm{~kg} / \mathrm{m}^{2}$ ), and received thyroid hormone because of chronic thyroiditis. The
ECG showed anterolateral ST elevation and reciprocal ST depression in inferior leads, consistent with an acute anterolateral MI. Laboratory data showed $10.8 \times 10^{3} / \mathrm{mm}^{3}$ leukcocytosis without an increase of creatine kinase. Her platelet count was raised at $69 \times 10^{4} / \mathrm{mm}^{3}$ and her haemoglobin was $10.2 \mathrm{~g} / 1$.

Three hours after the onset of back pain, the patient was taken to the catheterisation laboratory. Coronary angiography revealed a filling defect on the ostial portion of the right coronary artery (fig 1A). The left anterior descending (LAD) coronary artery was occluded at the mid-portion (fig 1B). She was treated initially with intracoronary tissue plasminogen activator $\left(640 \times 10^{4} \mathrm{IU}\right)$. This resulted in TIMI 1 flow in the LAD. Angioplasty was performed using a $3 \mathrm{~mm}$ Scuba balloon catheter through a $7 \mathrm{~F}$ guiding catheter (Advanced Cardiovascular Systems Inc, California, USA) and a 0.014 " flexible guide wire, but caused coronary dissection. Then $3.5 \times 18 \mathrm{~mm}$ gfx stent (Arterial Vascular Engineering, CA, USA) was successfully implanted. The occluded lesion improved significantly, but the perfusion delay in the LAD remained (no reflow, fig 1C). After these procedures, repeat angiography showed disappearance of the filling defect in the right coronary artery. Before the procedure, the patient received aspirin $(81 \mathrm{mg})$ and ticlopidine $(100 \mathrm{mg})$. Heparin $\left(1 \times 10^{4} \mathrm{IU} /\right.$ day $)$ and low molecular weight dextran $(100 \mathrm{ml} / \mathrm{h})$ were administered during and after the procedure. Peak serum creatine kinase was 4760 IU with an MB isozyme level of $483 \mathrm{IU}$.

The platelet count rose to a maximum of $113.8 \times 10^{4} / \mathrm{mm}^{3}$ two weeks after admission, without an initial drop. Oral busulfan was administered and then the platelet count fell to around $60 \times 10^{4} / \mathrm{mm}^{3}$ (fig 2). Peripheral blood smear evaluation revealed clumping giant platelets with heterogeneous morphology. Bone marrow examination showed numerous large hyperploid megakaryocytes. Normal myelopoiesis and erythropoiesis were present. Cytogenetics were normal and no Philadelphia chromosome was detected.

At repeated angiography one month after the procedure, there were no thrombus-like filling defects or significant athelosclerotic lesions in the coronary arteries, and the coronary flow in 

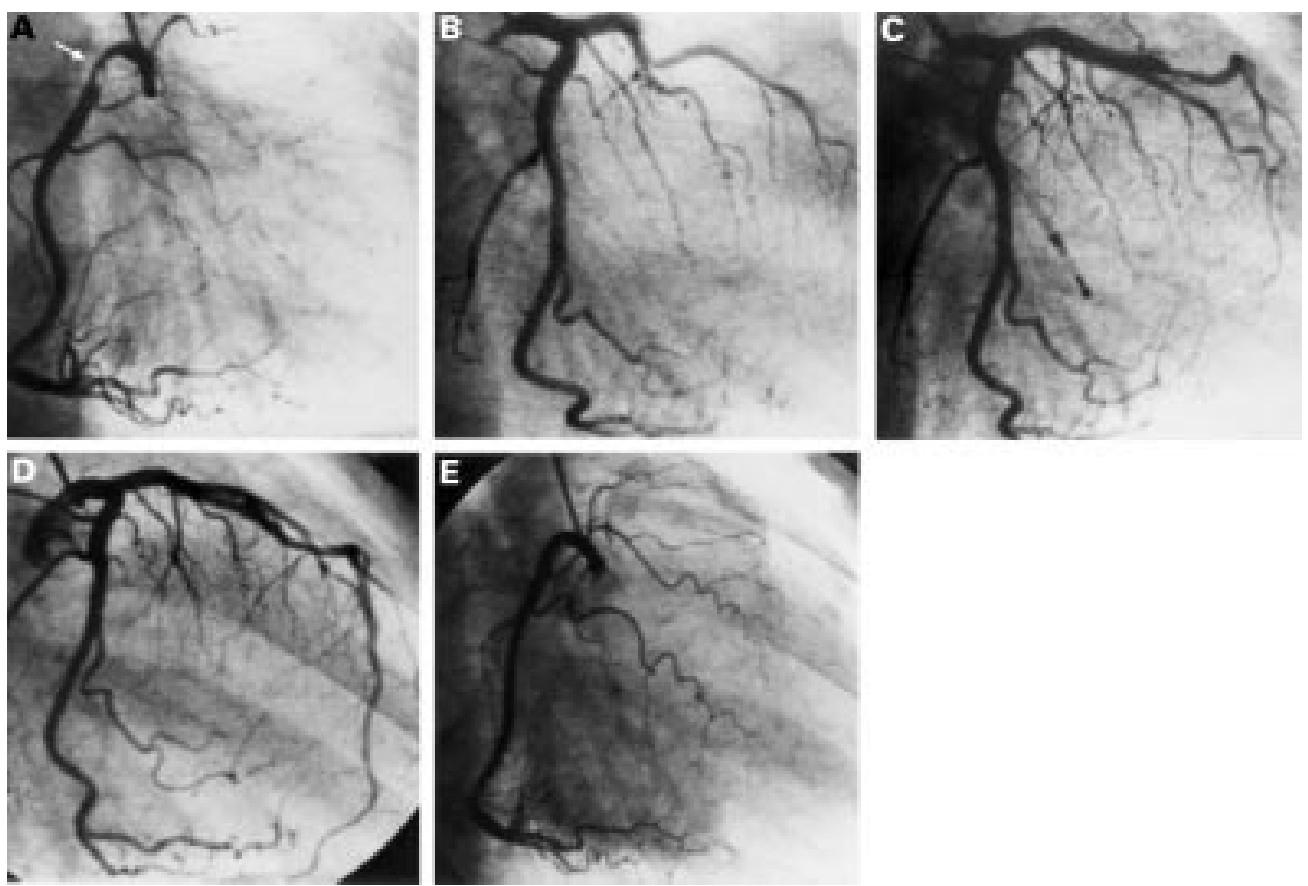

Figure 1 (A) and (B): Emergent angiography of right coronary artery (RCA) and left coronary artery (LCA). Arrows indicate thrombus-like filling defect with a hazy appearance in the proximal portion of RCA and completely occluded left descending artery (LAD). (C):LCA angiography after angioplasty. Though the occluded lesion was successfully dilated, the perfusion delay in LAD remained (no reflow: arrow). (D) and (E): Repeat angiography one month after infarction. There were no thrombus-like filling defects or significant athelosclerotic lesions in the coronary arteries, and the coronary flow in $L A D$ was not slow.

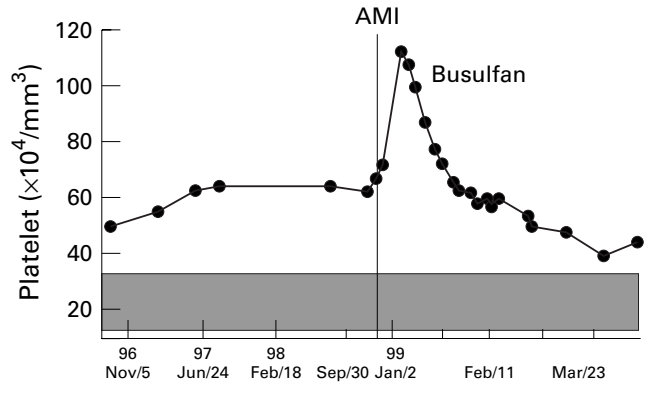

Figure 2 Time course of blood platelet counts in the patient. AMI indicates the onset of acute myocardial infarction. The shaded area shows the normal range of the platelet count in our institute.

LAD was not slow (fig $1 \mathrm{D}$ and E). Left ventriculography showed anteroapical hypokinesis with left ventricular ejection fraction of $68 \%$. She was discharged, without chest pain, with a platelet count of $60.6 \times 10^{4} / \mathrm{mm}^{3}$ and on aspirin (81 mg/day), ticlopidine (200 mg/day), and busulfan ( $2 \mathrm{mg} /$ day). She developed no major bleeding complications.

\section{Discussion}

ET has clinical tendencies to both haemorrhage and thrombosis. However, coronary thrombosis and acute MI have been observed rarely in ET compared with other myeloproliferative disorders (for example, polycythaemia vera). ${ }^{5}$

Several theories have been postulated for the cause of coronary thrombosis from thrombocytosis. These are: abnormal activation of the fibrynolytic system, enhanced platelet procoagulant activity, and increased plasma viscosity. ${ }^{3}$ Previous studies showed that platelet size, rather than the absolute platelet count, is correlated with thrombotic complications. In our patient, peripheral blood smear evaluation revealed clumping giant platelets although the initial platelet count was only moderately raised. Fagher et al described patients with acute MI who had an initial drop in platelet count followed by an increased and even thrombocytosis. ${ }^{8}$ They reported that in $25 \%$ of acute MI patients the platelet count increased by almost $70 \%$ three weeks after the onset. The transient rise of the platelet count in our patient after admission might be reactive, but there was no initial drop and the rise was over $100 \%$. Therefore, it is possible that the platelets were more haemostatically active at the onset of coronary thrombosis.

The aggressive inhibition of platelet aggregation and production may play an important role in treating ET associated ischaemic syndromes with coronary thrombosis. Michaels et al used a glycoprotein $\mathrm{IIb} / \mathrm{III}$ a receptor inhibitor, abciximab, for acute MI due to ET. ${ }^{4}$ However, the use of antiplatelet agents in these patients has been suspected of inducing haemorrhage rather than preventing thrombosis. Rossi et al showed that a low dose of aspirin can reduce the coronary thrombosis without increasing bleeding complications. ${ }^{5}$ Our patient did not develop major haemorrhagic complications during treatment with aspirin and ticlopidine, probably because of the low dosage adopted. There are also recent data from randomised clinical trials showing hydroxyurea to be effective in preventing ET related thrombotic complications by keeping the platelet count $<60 \times 10^{4} / \mathrm{mm}^{3}$. ${ }^{9}$ In our patient, busulfan was used to reduce platelet production. 
Our patient also had no reflow phenomenon after recanalisation of the LAD. Several mechanisms have been advocated for the no reflow phenomenon in animal models, and a major candidate is direct ischaemic microvascular injury. ${ }^{10}$ Our patient had acute MI with angiographically normal coronary arteries. The obstruction of LAD does not appear to be due to the rupture of atherosclerotic plaque, but is likely to be transient thrombosis. Therefore, it is possible that multiple microvascular thrombi contributed, at least in part, to the no reflow phenomenon in our patient.

In conclusion, we recommend long term prophylaxis with antiplatelet agents in patients with ET, even if the platelet count is only moderately raised. Cytoreductive treatment in combination with antiplatelet agents may be effective for the second prevention when thrombotic complications occur.

1 Randi ML, Fabris F, Stocco F, et al. Multiple, relapsing with thrombocytosis. Blood Coagul Fibrinolysis 1990;1:331-2.
2 Tai YT, Yu YL, Lau CP, et al. Myocardial infarction complicating postsplenectomy thrombocytosis, with early left ventricular mural thrombus formation and cerebral embolism — a case report. Angiology 1993;44:73-7.

$3 \mathrm{Koh} \mathrm{KK}$, Cho SK, Kin SS, et al. Coronary vasospasm, multiple coronary thrombosis, unstable angina and essential thrombocytosis. Int 7 Cardiol 1993;41:168-70.

4 Michaels AD, Whisenant B, MacGregor JS. Multivessel coronary thrombosis treated with abciximab (ReoPro) in a patient with essential thrombocythemia. Clin Cardiol 1998; 21:134-8

5 Rossi C, Randi ML, Zerbinati P, et al. Acute coronary disease in essential thrombocythemia and polycythemia vera. F Intern Med 1998;244:49-53.

6 Virmani R, Popovsky MA, Roberts WC. Thrombocytosis, coronary thrombosis and acute myocardial infarction. $A m \mathcal{F}$ Med 1979;67:498-506.

7 Martin JF, Trowbridge EA, Salmon GL, et al. The biological significance of platelet volume: its relationship to bleeding time, platelet thromboxane B2 production and megakaryocyte nuclear DNA concentration. Thromb Res 1983;32:443-60.

8 Fagher B, Sjogren A, Sjogren U. Platelet counts in myocardial infarction, angina pectoris and peripheral artery disease. Acta Med Scand 1985;217:21-6.

9 Cortelazzo S, Finazzi G, Ruggari M, et al. Hydroxyurea for patients with essential thrombocythemia and a high risk of thrombosis. N Engl f Med 1995;332:1132-6.

10 Ishihara $M$, Sato $H$, Tateishi $H$, et al. Attenuation of the no-reflow phenomenon after coronary angioplasty for acute myocardial infarction with intracoronary papaverine. Am Heart f 1996;132:959-63. 